\title{
Research of the possibilities for using and linking TRIZ Methods with Systems Engineering
}

\author{
Ovidiu Bielefeld ${ }^{1}$, Vladimir Sizikov ${ }^{2}$ und Dr. Nadine Schlüter ${ }^{1}$ \\ ${ }^{1}$ University of Wuppertal, Gaußstraße 20, 42119 Wuppertal, Germany \\ ${ }^{2}$ Robert Thomas Metall- und Elektrowerke GmbH \& Co. KG, \\ Hellerstraße 6, 57290 Neunkirchen, Germany
}

\begin{abstract}
Increasing complexity and global competition are forcing product development to develop faster products that are marketable and innovative. Thereby, key issues are increasing product functionality and user-friendliness. The significant engineering contradiction is: How can user-friendliness of the products be optimized for the customer without adversely affecting the functionality of the product? This requires a systematic consideration of the interactions between the customer and the product as well as the effects and interactions of the functions within a product. TRIZ provides a variety of methods to analyze and solve problems, incl. resolving of engineering contradictions. In addition, an interdisciplinary and transparent model can be created with ModelBased Systems Engineering (MBSE) to improve product understanding.

The scope of this research is to combine the potential of MBSE with TRIZ in order to generate an optimal approach regarding implementation and networking from TRIZ function models in to a MBSE overall model. Based on the MBSE overall model both usability and system functionality (especially harmfull function) should be considered.

Keywords: TRIZ, Model-Based Systems Engineering (MBSE), system model, vacuum cleaner, engineering contradiction, function analysis, function model
\end{abstract}

\section{Introduction}

In product development it is important not only to focus on its functionality or primary useful function(s) (PNF) according to TRIZ, but also to deal with the context of application and handling to improve the ergonomy and usability of products. Due to the technological development the complexity of products is increasing significantly.Usability is a topic of many scientific researches and practical efforts. Especilly TRIZ has powerful tools for analysis and understanding of developmnent problems. Also very complex problems can be handled with both classic and advanced TRIZ tools. A further alternative solution in product development is provided by the MBSE. Because only when the product is understood as a model, the complexity can be made tangible and manageable [1]. Based on the principles of the MBSE, interactions and dependencies between different functions and components within a system can be displayed transparently. Furthermore, it is theoretically possible to model several (sub) models (e. g. function models) from TRIZ in an overall model or a super system 
that can serve as the basis for a common understanding within a multidisciplinary team. Consequently, MBSE is very suitable as a supplement forTRIZ. The goal of this research is to combine the potential of MBSE with TRIZ in order to generate an optimal approach regarding implementation and networking from TRIZ function models into a MBSE overall model. Based on the MBSE overall model both usability and system functionality (especially harmfull functions) should be taking into account. In order to explain how the approach was developed, in section 2 selected methods and tools of TRIZ will be presented. Section 3 gives a short introduction to MBSE. This is to better understand the approaches presented in Section 4 for the combinations of TRIZ and MBSE. The new approach is presented using the vacuum cleaner as an example. Finally, the results in the last section are discussed and an outlook on the further research work is given.

\section{TRIZ methods}

\subsection{Problem analysis with classical TRIZ}

Classic TRIZ includes the methods for clarifying the task, determining the functions and abstraction of the problem as well as finding a solution with various tools [2].

Function modeling is used to analyze the functioning of a technical system. It defines a target component (from the Super System) that is served with the primary useful function of a technical system [3]. The problem analysis focuses only on this target component. In the problem analysis for consumer goods, the authors have discovered a special feature. A an engineering system addresses two target components from the Super System:

1. An item treated by the given system (main function of the system).

2. The human, who uses the given system (customer experience).

As a case study, the vacuum cleaner was taken. Formulation of the primary useful functions, which occur at the same time, is shown in Fig. 1.

In this illustration, the vacuum cleaner is mapped as a component with all subsystems. Details on the case study, including subsystem components as well as useful and harmful functions follow in Chapter 4.

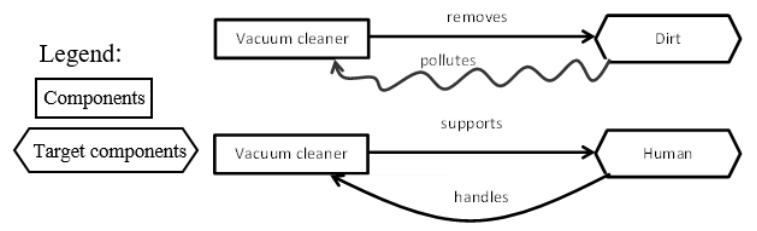

Fig. 1. Target components for vacuum cleaners as a technical system

In the interaction between vacuum cleaner and dirt, dirt exerts a harmful function on the vacuum cleaner. But the function "removes" is not affected. Harmful effects of the function "pollutes" appear in the other function model by the interaction between human and vacuum cleaner (preparation, vacuuming, and emptying/cleaning). There- 
fore, the dirt increases the operating effort. Though the vacum cleaner supports the human in various ways. For example ergonomically (the person does not have to bend down), health-wise (no inhalation of dirt) and saves time (little effort). In the example shown, two function models are to be constructed, whereby some functions from one model influence the functions of the other model. Due to the fact that the use of the vacuum cleaner by humans displays a process with three phases, as described above, the TRIZ Function-Analysis for processes [4] is relevant for the case study

The process steps are also called use case processes. Use case is defined as the descrition of an intended (or existing) use process that describes the user's handling of the system [5].

Also, in the process analysis for the case of the vacuum cleaner two target components are relevant: dirt and human. This results in two different processes:

1. The use case processes of dirt handling (remove from the ground, suck in, catch in the filter system, etc.)

2. The use case processes of handling the device by a human (add filter medium, assemble, operate and clean the device).

The following difficulties have come with the problem analysis by means of the described tools of the classical TRIZ:

1. Simultaneous addressing of two target components. These two target components appear in two different functional models. The problems of individual models are interlinked with other models.

2. A special challenge for the functional analysis is to identify the customer experience (soft and emotional factors).

The tools of the classic TRIZ were used in the project (case example vacuum cleaner). The core problems (engineering contradictions), however, could not be identified due to the problem complexity. Therefore, further research was done in order to analyse further development of the TRIZ methodology. The results of this analysis will be described in the next section.

\subsection{Further research for TRIZ-based problem analysis}

As described in section 2.1, applications of the tools of the classical TRIZ in analyzing the problems with the focus in the field of customer experience encounter several difficulties. In science and practice through further development of the TRIZ methodology (so-called Advanced TRIZ), various tools have emerged for the analysis of complex questions. This section discusses their relevance. After the research and preselection, the authors considered the following tools from Advanced TRIZ:

1. OTSM-TRIZ (Russian acronym for General Theory of Powerful Thinking)

2. IDM (Inventive Design Method)

3. FA+ (Function Analysis Plus)

4. Cause Effect Chain Analysis (CECA)

As summarized by [6], OTSM consists of the following elements: problem technology, partial solution technology, contradiction technology, and problem flow technology. Based on these four elements, a network of problems is developed, which is used 
to describe the actual state. The graphical representation of problems and partial solutions help to identify the main engineering contradictions. The strength of OTSM is the simultaneous consideration of problems and solutions as well as building the causalities between individual elements in the network of problems.

IDM Methodology [7] has further deepened and automated the treatment of problem networks. The problem analysis with IDM creates a problem graph. Through structured syntax and algorithmic evaluation of existing relationships, it is possible to automatically extract the contradictions from the problem graph. The methodology also takes up a search for solutions. Thus, the tracing of the contradictions eliminated by a solution is possible. Also, change from a problem graph is representative (transition of a model of problem into the model of solution).

FA+ is another method in consideration of existing research. In the work of Lee [8], the TRIZ function modeling is combined with the Inventive Standards and CauseEffect Chain Analysis. The objective here was the synthesis of a practical and systematic method for simplifying the search for solutions. FA+ uses classic functional modeling with the help of innovative standards to detect causalities and temporal relation between individual components and functions. The advantage of this method is a combination of problem models (functional analysis) and solution models (innovative standards) in one presentation.

Cause Effect Chain Analysis (CECA) is another TRIZ-tool for problem analysis. It would be also possible to visualize the dirt handling and the process of device handling with the help of CECA. But CECA treats only disadvantages or negative effects of the system as nodes [3]. So modelling with CECA would bring the same disadvantages as a function model. Furthermore CECA deals with negative effects of useful functions only. That's why the authors didn't use CECA in the project.

The four described approaches from Advanced TRIZ have the following features compared to classic tools:

1. A higher degree of abstraction in problem formulation.

2. Consideration of possible solutions already in the problem description.

3. Systematic detection of contradictions (or standard solutions).

4. A visualization that allows detection of one or more critical problems. At the same time, the problems are also prioritized.

This state of research has given important directions for problem analysis in customer experience. These are, among other things, consideration of all problems of the technical system in one model, search for algorithms and automated problem analysis, and quick integration of the solution search in a model. Nevertheless, based on the findings from classical and advanced TRIZ in relation to the scope of this paper following issues still remain unanswered:

1. How interact two or more target components from two or more function models (problem model) with each other? In the case of customer experience, this is at least one target component of the technical function (e.g., dirt, see 2.1) and the human being, as a user of a technical system.

2. How use case processes can be taken into consideration in a same model? This means that the relationships between components and functions happen in vari- 
ous use case processes (e.g. one of the use case process of the vacuum cleaner is "dirt suction").

3. How contradictions between compontens, useful function and harmful function in a same model with technical parameters (e.g., filter effect) can be taken into consideration in a same model?

Those issues could be solved by a new approach based on TRIZ and MBSE. Using MBSE a overall model can be created in order to map two or more target components from two or more function models. Moreover, use case processes could also be considered in the same model. And also contradictions between compontens, useful functions and harmful functions could be shown. To meet these goals, MBSE has been examined to detect its potential (section 3). Finally, a new approach based on TRIZ and MBSE has been developed and tested using a case study (section 4).

\section{Systems Engineering, Model-Based Systems Engineering and Demand Compliant Design}

To master the complexity of products it is helpful to understand the product as a system. This can be done on the basis of a holistic and transdisciplinary model according to the basic principles of MBSE [1]. In addition a system model forms the basis for a common understanding of system behavior within a multidisciplinary team $[1,9]$. The model enables a systematic identification of the dependencies between different system elements (e.g., system component, target component, functions, etc.).

This systemic thinking from MBSE arose from Systems Engineering (SE), which already has been used in the aerospace industry in the 1950s [10]. SE is defined as "an interdisciplinary approach and should make the development of systems methodically possible. SE focuses on a holistic and collaborative understanding of stakeholder requirements, discovery of solution options and documentation of requirements as well as synthesizing, verifying, validating, and developing of solutions. The whole problem is considered from concept development to system development and the SE provides suitable methods, processes and best practices for this "[11]. As an interdisciplinary discipline, SE acts as a "link" between the different disciplines [12].

The model-based implementation of the SE is the MBSE. Through the MBSE, a system model can be created. There are different modeling languages and approaches in MBSE (e.g., SysML, Modelica or CONSENS), but for this work and due to the positive experiences several years of project work from the Demand Compliant Design (DeCoDe) approach is selected. DeCoDe is an approach for a standardized description to model technical systems under the principles of systematic thinking and acting [1]. Modeling with DeCoDe is matrix based i.e. data and information about the system is recorded in a structured way and systematically reproduced. To analyze the system it can be examined based on "classic views" of product development: Requirements, Components, Functions and Processes. These views are linking goaloriented by using the DeCoDe Main matrix within the DeCoDe-Tool (see Fig. 2). 


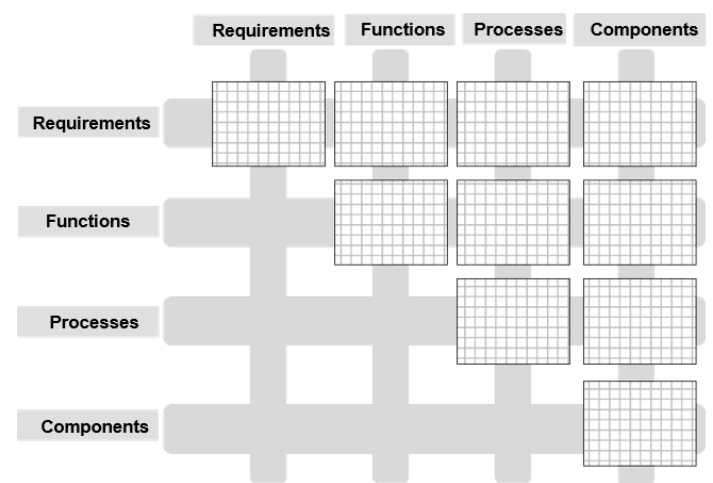

Fig. 2: The Main matrix of DeCoDe with its 4 views [in compliance with 14]

The complete linking of views makes a multidimensional view of the entire system possible. This is realized by the so-called Multi-Domain Matrix (MDM). Basically, in matrix-based modeling, there are three types of matrices:

- Domain Structure Matrix (DSM): describes the interlinking within a view (e.g., within the components-components matrix the components structure can be described.)

- Domain Mapping Matrix (DMM): describes the interlinking between two views (e.g., the components-processes matrix describes the relationship between the components and processes)

- Multi-Domain Matrix (MDM): describes a multi-dimensional view of the entire system by mapping all views with their system elements and relations [13].

For the implementation of the DeCoDe approach, the complexity management software Loomeo® is used. The software not only enables the presentation of the DeCoDe data, but also the graphical visualization of the DeCoDe element types and their relations (see Fig. 3). By focusing on an element and its relations, individual cause-and-effect relationships can be discussed as part of workshops in the interdisciplinary development team through various other elements related to the focused element, as Figure 3 shows.
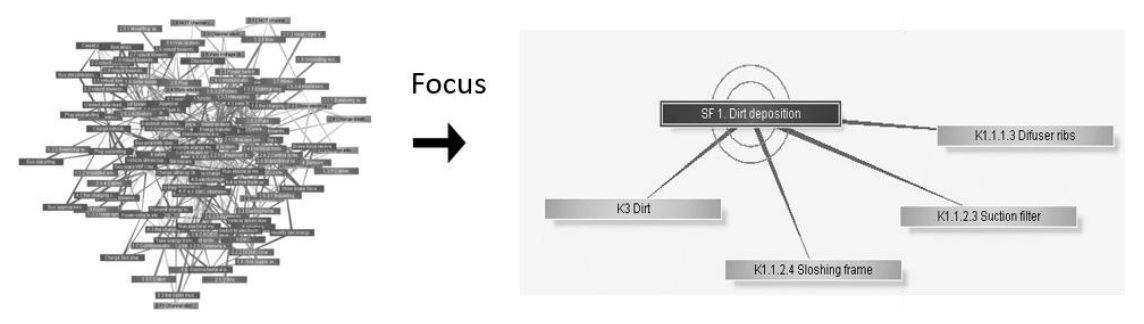

Fig. 3: Exemplary visualization of the identification of cause-and-effect relationships for individual elements through focusing by using Loomeo® function "Environment's Fokus" [Principle diagram]. 
By selecting a system element and using the Loomeo ${ }^{\circledR}$ "Environment's Focus" feature can bee seen which elements are interacting with each other. For example, in case of the failure of the selected element, it will be obvious via the corresponding relations which components would be affected directly or indirectly. The relationships and dependencies identified in this way are stored separately with a unique ID. This information from the system model can be used specifically for different TRIZ methods from the phases of problem analysis processes and problem-solving processes. The knowledge transfer between MBSE and TRIZ methods is shown in the next section.

\section{Investigation of possibilities for combinability between TRIZ methods and Systems Engineering}

The goal of this study is to combine the potential of MBSE with TRIZ in order to generate an approach regarding implementation and networking from TRIZ function models into overall model based on MBSE.

The chapter is divided into three subchapters, which together represent the new approch for combination of TRIZ methods and MBSE (see Fig. 4). The first phase contains the classic functional model according to TRIZ. In this phase, according to TRIZ and to the methods presented in section 2, the functional model is created. Building on the first phase, the functional model according to TRIZ is extended by using the tools and the procedure from MBSE, which were presented in section 3. The third phase contains an analysis and a targeted search for problems and causalities in the context of the entire system model. By means of the focus function from section 3 relationships and dependencies of functions and components will be identified. Finally, the findings from the analysis will be presented. The focus here is to show what concrete benefits the MBSE approach has for TRIZ. The approach will be explained in more detail with by showing the vacuum cleaner as application example.

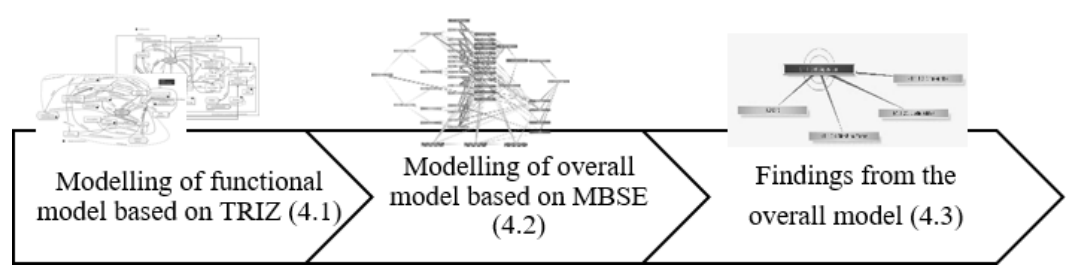

Fig. 4: Approch for the combinability between TRIZ methods and SE

\subsection{Modelling of functional model based on TRIZ}

THOMAS vacuum cleaners have special filtration technologies. Aqua-Fresh-AirBox uses water as a filtration medium. The advantages for target groups are the following:

1) Humidification of dust and capture even fine particles inside the filtering unit. This function is very important for allergic sufferers. 
2) Water can also bind smell, odors and formaldehyde. It is an advantage for households with cats and dogs.

The technical function (filtering) does not have any problems. Far over $90 \%$ of all dirt particles are catched within the Aqua-FreshAir-Box. Due to the fact that the contaminated water cannot be kept for a long time, there is a need to wash out the dirt after using of the vacuum cleaner. By collecting the information from different sources (promoters, market research, online reviews) the project team identified the demand for improving of customer experience with the filtration unit. It refers to the handling of vacuum cleaner: preparation, vacuuming (collecting the dirt) and cleaning of the filtration unit.

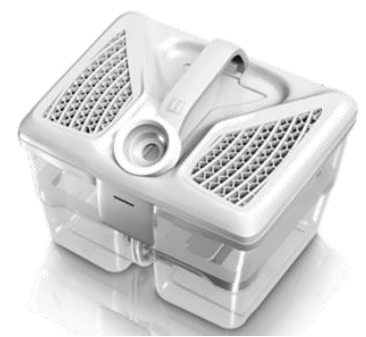

Fig. 5: Aqua-FreshAir-Box as a component form the vacuum cleaner system

As described in the section 2.1, there are two targets components for the given engineering system: dirt (technical function - filtering) and human (usability, handling, etc.). Follow-ing steps were done with TRIZ, addressing the problem of customer experience:

1) innovation checklist,

2) function analysis with dirt as a target component,

3) function analysis with human as a target component,

4) flow analysis for materials: air, dirt (which can be differed from fine dust and coarse dirt, incl. hairs).

Both function models (see Fig. 6), which are created according to classic TRIZ, describing respectively filtering and handling, were complicated. There are 13 components of the engineering system, having multiple useful and harmful functions.
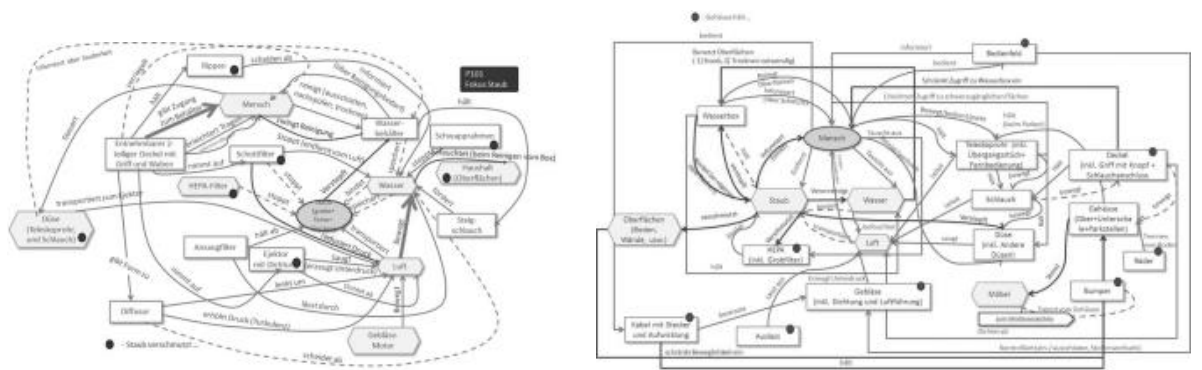

Fig.6: Exemplarly visualization of both functional models (left - dirt as a target component, right - human as a target component) [Principle diagram]. 
As mentioned in the section 2.1, several useful and harmful functions from one model have influence at the another model. These models were shown as examples, an entire presentation would be too unclear. In addition, the data is confidential and cannot be published. Furthermore, these functions models serve as a basis for one overall model which is created with MBSE.

\subsection{Modelling of overall model based on MBSE}

The extended modelling begins with the transfer of the function model (see Fig. 6) from section 4.1 into the MBSE overall modell. In addition to the functions and the components, the usage processes are also integrated into the system model. The meshing is template-based according to the principles and using the DeCoDe main matrix, which were presented in section 3 . The graph below outlines a schematic representation of the components hierarchy (as well as target components Human and Dirt), the useful functions (as well as a harmful function) and the three processes involving the components (see Fig. 7). Based on this graphical representation, the overall system or the Super System to be analyzed can be viewed in a structured manner. Furthermore, several functional models based on TRIZ can be transferred into this one overall model, so that just a single model is available for multiple problem analysis. The functions, whish are interlinked with the components, could be the same but also similar.

Based on the extended model, the next phase will analyze the problem according to the TRIZ principles.

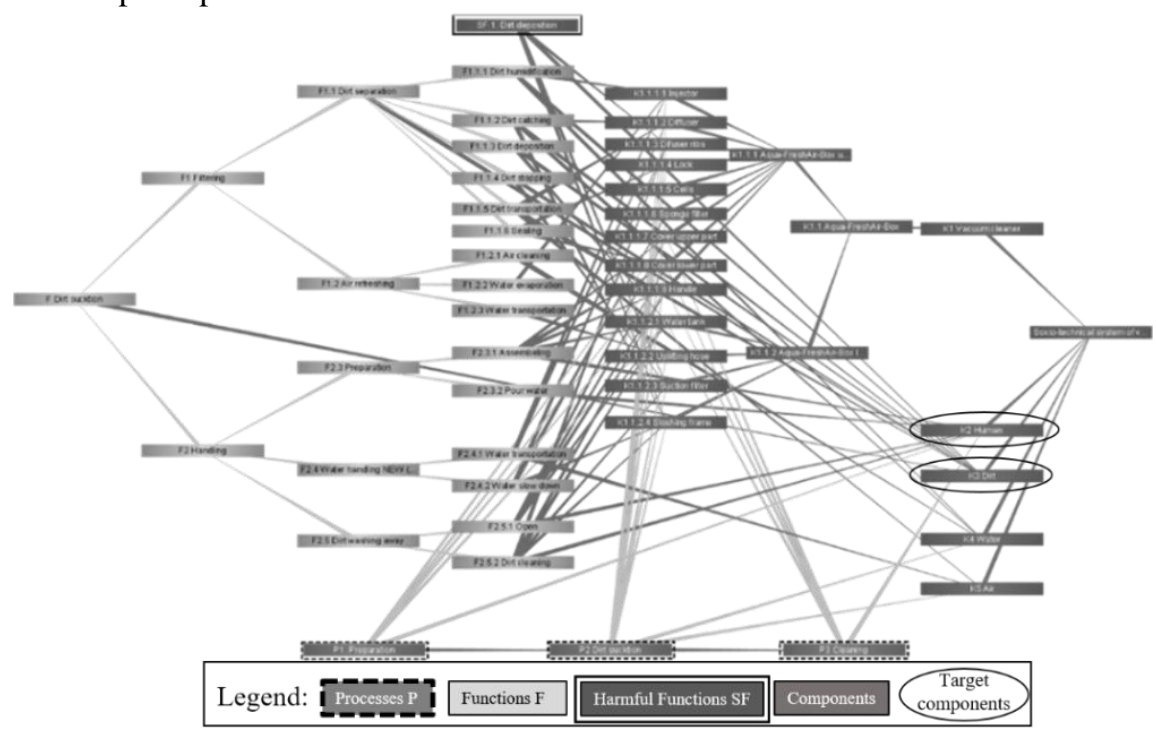

Fig. 7. Schematic representation of the extended model according to Systems Engineering with useful functions, harmful functions, components, target components and use case processes [Principle diagram]. 


\subsection{Findings from the overall model}

The analysis begins based on the TRIZ methodology, i.e. with the problem search or problem definition [3]. For this purpose, the overall model from section 4.2 is used as well as the focus function for a specific search for possible problems and causalities within the Super System. First the focus function was used for the target component Human, then the focus will be put on the harmful function "Dirt deposition". Using the focus function and spatial separation provide a quick way to identify the components that perform the harmful "Dirt deposition" function (see Fig. 8).

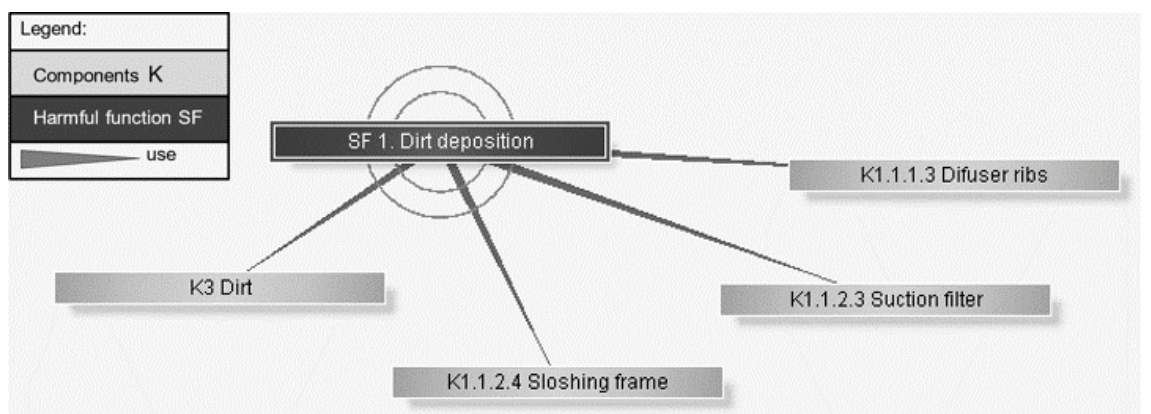

Fig. 8. Correlation between the harmful function of "Dust deposition" and the components that implement this function (e.g. Suction filter, Sloshing frame and Diffuser ribs)

Thus, it was thus possible to identify that the components Suction filter, Sloshing frame and Diffuser ribs and the target component "Dirt" are involved in this harmful function. At the same time, it was found that the components "Diffuser ribs" and "Sloshing frame" also realize the useful function of "Water slow down" (see Fig. 9).

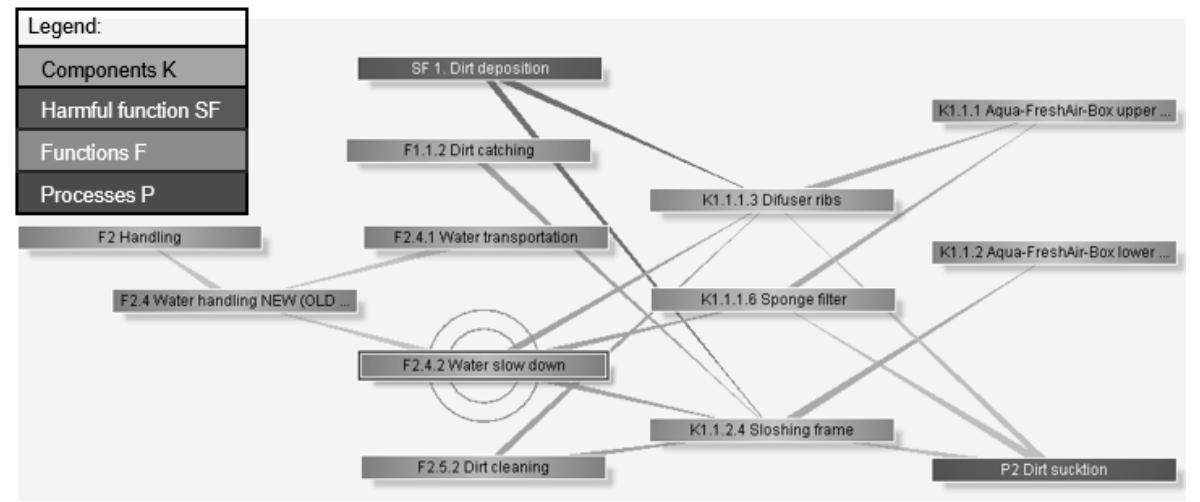

Fig. 9. The components Sloshing frame and the Diffuser ribs realize the useful function "Water slow down"

In this way, the engineering contradiction could be identified. Furthermore, it has been focused on the component "Suction filter" to find out which useful functions this component implements in the model (see Fig. 10). 


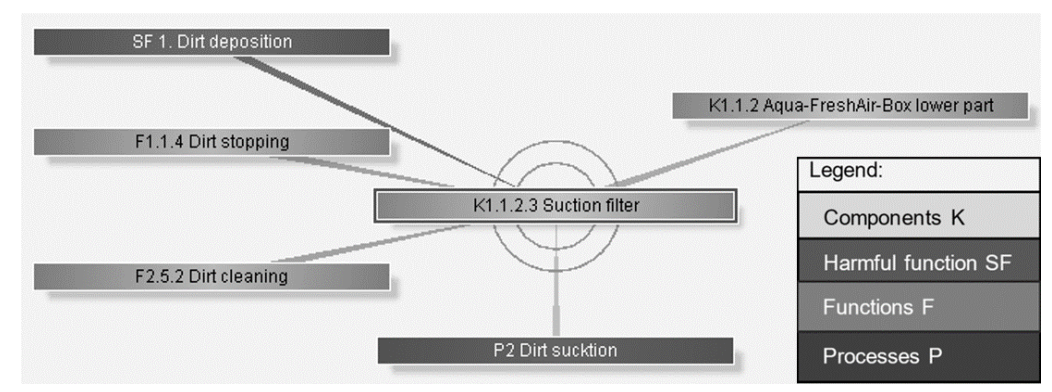

Fig. 10. The component Suction filter realize the useful function "Dirt stopping" and at the same time the harmful function "Dirt deposition"

The figure shows that the Suction filter fulfills the useful function of "Dirt stopping" as well as the harmful function of "Dirt deposition" simultaneously. In addition, the "Suction filter" is involved in the "Dirt suction" process and is a sub-component of the Aqua-Fresh-Air-Box (see Fig.10). The involvement of the component "Suction filter" in one useful function and one harmful function forms this engineering contradiction in the model (parameter of suction filter is the size of the grid cell):

IF the suction filter has a big grid cell, THEN it does not deposit the dirt, BUT it does not stop the dirt. The inverse engineering contradiction is: IF the suction filter has a small grid cell, THEN it stops the dirt, BUT it deposits the dirt.

A physical contradiction here is: Grid cell must be big and small. Identification of engineering and physical contradiction with classical function modelling (section 4.1) was not possible. The useful function "Dirt stopping" belongs to the model with dirt as a target component (filtering). While the harmful function "Dirt deposition" appears at the model with human as a target (usability). The identification of the two enginering contradictions provides a good basis for a well-founded problem definition and the continuation of the problem-solving process according to TRIZ. In the same way the model described in the chapter 4.2 enabled identification of further engineering contradictions.

\section{$5 \quad$ Conclusion and outlook}

The goal of this research paper was to combine the potential of MBSE with TRIZ in order to generate an optimal approach regarding implementation and linking from TRIZ function models in to a MBSE overall model. Furthermore, the overall model to detect the impact between usability (use case processes) and the system functionality (especially harmfull function).

It can be shown that tools from MBSE are useful for the TRIZ analysis methods in order to transparently depict relationships, dependencies, and causalities (see 4.3). Using MBSE a overall model was created. The model allows an efficient and quick search for problems (TRIZ problem-solving process) and formulation of both engineering and physical contradictions. In addition, several target components and functions are mapped in a transparent model. Also the hierarchic structure of functions 
according to MBSE allows fast identification of components, having same or similar functions.

However, this research is at the beginning and many more investigations remain open. Further research is planned, such as the implementation of a quantitative evaluation by means of the software in the analysis, so the components and functions as well as their relations can be weighted. Furthermore, it is necessary to investigate whether a change in the procedure of the analysis, e.g. focus on the use case process (useability) and not on the harmful functions, leads to different results. These aspects could form an integral part of further research into the combinability between TRIZ and SE.

\section{References}

1. Winzer, P.: Generic Systems Engineering - Ein methodischer Ansatz zur Komplexitätsbe-wältigung. Springer Vieweg Verlag, (2016).

2. VDI-Standard: VDI 4521 Blatt 1, VDI, Dusseldorf, (2016).

3. VDI-Standard: VDI 4521 Blatt 2, VDI, Dusseldorf, (2018).

4. MATRIZ Homepage, https://matriz.org/, last accessed 2019/03/28.

5. Jacobson, Ivar: Object-oriented development in an industrial environment. In: Norman Meyrowitz (Hg.): OOPSLA '87 conference proceedings. Object-oriented programming systems, languages and applications. S. 183-191. New York, N.Y.: ACM (SIGPLAN notices spec. iss, 22,12), (1987).

6. Nagel, T.: Application of OTSM-TRIZ for definition of Engineering Process model and re-lated deployment Strategy. In: Papers Presented at the 14th International TRIZ Future Con-ference - Global Innovation Convention, Lausanne/CERN, 29.-31.10.2014, pp. 57-60, ETRIA e. V., (2014).

7. Cavallucci, D.; Fuhlhaber, S.; Riwan, A.: Assisting decisions in Inventive Design of com-plex engineering systems. In: Procedia Engineering, Volume 131, Pages 975-983, Sci-enceDirect, (2015).

8. Lee M.-G.: How to Generate Simple Model Solutions Systematically from Function Analy-sis Diagram. In: Selected papers presented at the 16th International TRIZ Future Conference, October 24-27, 2016, Wroclaw, Poland, pp. 42-47, ETRIA e. V., (2017).

9. Haberfellner, R. ; Fricke, E.; de Weck, O.; Vössner, S. (2012): Systems Engineering Grundlagen und Anwendungen Orell Füssli Verlag Zürich, (2012).

10. Gausemeier, J.; Dumitrescu, R.; Steffen, D.; Czaja, A.; Wiederkehr, O.; Tschirner, C.: Sys-tems Engineering in der industriellen Praxis (Studie), wentker druck $\mathrm{GmbH}, \mathrm{Pa}$ derborn, (2013).

11. INCOSE Systems engineering handbook - A guide for systems life cycle processes and ac-tivities. Wiley, Hoboken, New Jersey, (2015).

12. Weilkiens T: Systems engineering with SysML; Modeling, analysis, design. Morgan Kaufmann OMG Press/Elsevier, Amsterdam, (2007).

13. Lindemann, M. Maurer and T. Braun, "Structural Complexity Management," SpringerVerlag, Berlin Heidelberg, (2009).

14. Winzer, Petra; Sitte, Joaquin: Demand Compliant Design. Accepted for publication in IEEE Transactions on Systems, Man and Cybernetics Part A, Volume 41, (2011). 